\title{
Evoluções e repercussões de um ano de Sars-cov2 no Estado do Pará, Brasil
}

\author{
Evolutions and repercussions of one year of Sars-cov2 in the State of Pará, Brazil \\ Evoluciónes y repercusiones de un año de Sars-cov2 en el Estado de Pará, Brasil
}

Maria Jaciele de Jesus Matos ${ }^{1}$, Taynara da Costa Silva ${ }^{2}$, Isabela Pantoja da Cruz ${ }^{3}$, Dayse Vanessa Araújo Neves ${ }^{4}$, Marildete da Conceição Paula ${ }^{5}$, Vanessa Ladyanne da Silva Costa ${ }^{3}$, Davi Silva Santana ${ }^{3}$, Letícia Gomes de Oliveira ${ }^{6 *}$.

\section{RESUMO}

Objetivo: Descrever as evoluções e repercussões de um ano após o primeiro caso de SARS-CoV-2 no Estado do Pará, Brasil. Métodos: Trata-se de um estudo epidemiológico-descritivo dos casos confirmados e óbitos por COVID-19 no Estado do Pará, no período de 1 ano. Resultados: Foram registrados 420.372 casos, sendo a maior prevalência de casos confirmados em indivíduos do sexo feminino $(53,4 \%)$, e óbitos em indivíduos do sexo masculino $(60,1 \%)$. Pode-se analisar os municípios com mais casos confirmados, evidenciando a capital paraense, Belém (20,45\%). Indivíduos adultos jovens apresentaram-se como os mais acometidos pela doença $(23,5 \%)$, no entanto, a prevalência dos óbitos foi mais elevada na faixa etária 70 a 79 anos (26,7\%), fazendo relação com às comorbidades com maiores índices, como as cardiopatias $(43 \%)$ e diabetes (33\%). Associada a essas conjunções, houve um crescimento da necessidade de cuidados intensivos $(87,63 \%)$. Conclusão: Acredita-se que com a identificação e esclarecimento dos dados epidemiológicos acerca da COVID-19, possibilita-se que haja uma reflexão sobre as condições e notificações dos casos no estado, sendo eficaz em retratar os índices de morbidade e mortalidade. Além disso, os indicadores dos casos confirmados no estado, demonstram a necessidade de cuidados redobrados, tanto por parte da população quanto das autoridades.

Palavras-chave: Coronavírus, Epidemiologia, Pandemia.

\section{ABSTRACT}

Objective: To describe the evolutions and repercussions of one year after the first case of SARS-CoV-22 in the State of Pará, Brazil. Methods: This is an epidemiological-descriptive study of confirmed cases and deaths by COVID-19 in the State of Pará, Brazil, in a period of 1 year. Results: There were 420,372 cases, with the highest prevalence of confirmed cases in females (53.4\%), and deaths in males (60.1\%). It is possible to analyze the municipalities with the most confirmed cases, evidencing the capital of Pará, Belém (20.45\%). Young adult individuals were the most affected by the disease (23.5\%), however, the prevalence of deaths was higher in the age group 70 to 79 years $(26.7 \%)$, related to comorbidities with higher rates, such as heart diseases (43\%) and diabetes (33\%). Associated with these conjunctions, there was an increase in the need for intensive care (87.63\%). Conclusion: It is believed that with the identification and clarification of epidemiological data about COVID-19, it is possible to reflect on the conditions and notifications of cases in the state, being effective in portraying morbidity and mortality rates. In addition, the indicators of confirmed cases in the state demonstrate the need for redoubled care, both by the population and the authorities.

Keywords: Coronavirus, Epidemiology, Pandemic.

\section{RESUMEN}

Objetivo: Describir las evoluciónes y repercusiones de un año después del primer caso de SARS-CoV-2 en el Estado de Pará, Brasil. Métodos: Se trata de un estudio epidemiológico-descriptivo de casos confirmados y muertes por COVID-19 en el Estado de Pará, Brasil, en un período de 1 año. Resultados: Hubo 420.372 casos, con mayor prevalencia de casos confirmados en mujeres (53,4\%), y defunciones en hombres $(60,1 \%)$. Es posible analizar los municipios con más casos confirmados, evidenciando la capital de Pará, Belém (20,45\%). Los individuos adultos jóvenes fueron los más afectados por la enfermedad (23,5\%), sin embargo, la prevalencia de muertes fue mayor en el grupo de edad de 70 a 79 años $(26,7 \%)$, relacionadas con comorbilidades con mayores tasas, como las cardiopatías $(43 \%)$ y diabetes (33\%). Asociado a estas conjunciones, hubo un aumento en la necesidad de cuidados intensivos (87,63\%). Conclusión: Se cree que con la identificación y esclarecimiento de los datos epidemiológicos sobre COVID-19, es posible reflexionar sobre las condiciones y notificaciones de casos en el estado, siendo efectivos en la representación de las tasas de morbilidad y mortalidad. Además, los indicadores de casos confirmados en el estado demuestran la necesidad de redoblar la atención, tanto por parte de la población como de las autoridades.

Palabras clave: Coronavirus, Epidemiología, Pandemia.

\footnotetext{
${ }^{1}$ Universidade da Amazônia (UNAMA), Ananindeua - PA.

2 Faculdade Estácio Castanhal (ESTÁCIO), Castanhal - PA.

${ }^{3}$ Universidade Federal do Pará (UFPA), Belém - PA.

${ }^{4}$ Faculdade Integrada Brasil Amazônia (FIBRA), Belém - PA.

${ }^{5}$ Centro Universitário Metropolitano da Amazônia (UNIFAMAZ), Belém - PA.

${ }^{6}$ Instituto Evandro Chagas (IEC), Belém - PA. *E-mail: gomes_15_letici@hotmail.com
} 


\section{INTRODUÇÃO}

Segundo Liu X, et al. (2020), a rápida disseminação do SARS-CoV-2 iniciou-se no período de dezembro de 2019, representando uma emergência de saúde pública mundial. O vírus atingiu 25 países em apenas um mês e a Organização Mundial da Saúde (OMS) declarou que a Coronavírus Disease (COVID-19) como pandemia (CAMPOS ACV e LEITÃO LPC, 2021).

O coronavírus pertence à família Coronaviridae, com RNA de fita simples, subdividido em 4 gêneros: alfa, beta, delta e gama. A abrangência clínica da infecção por coronavírus é muito ampla, podendo variar de um simples resfriado até uma pneumonia grave. A infecção por COVID-19 pode ser assintomática ou geralmente apresenta sinais e sintomas, incluindo problemas respiratórios leves e febre persistente, em média de 5 a 6 dias após a infecção, tendo em vista que o período médio de incubação de 5 a 6 dias, intervalo de 1 a 14 dias (LIU X, et al., 2020; LIMA FET, et al., 2020).

No início de fevereiro, o Brasil declarou a Emergência em Saúde Pública de Importância Nacional (ESPIN). O primeiro caso de COVID-19 no Brasil foi confirmado em 26 de fevereiro de 2020 e o primeiro óbito deu-se em 17 de março, desde esse momento a COVID-19 se disseminou rapidamente depois de quase dois meses resultando os casos de registro em mais de 9 mil óbitos (BRASIL, 2020; OLIVEIRA WK, et al., 2020; FRANÇA EB, et al., 2020).

O Ministério da Saúde (MS) passou a orientar a população o uso de máscaras de pano, para atuarem como barreira à propagação do SARS-CoV-2, além de medidas de segurança, como distanciamento social, etiqueta respiratória que consiste em cobrir a boca e o nariz ao espirrar e tossir, higienização das mãos e limpeza e desinfeção de ambientes (SOARES KHD, et al., 2021; BRASIL, 2020).

A situação da região norte tornou-se uma das mais alarmantes do país devido a discrepância socioeconômica da população, visto que todos os estados apontam indicadores de pobreza acima da média nacional. Dessa forma, essas populações ficam mais vulneráveis aos impactos de diferentes doenças, seja por dificuldades à rede de acesso, assistência em saúde e/ou acessibilidade (MENDONÇA FD, et al., 2020).

No estado do Pará a situação era gravíssima, principalmente devido à falência da porta de entrada ao sistema de saúde, o que pode ter resultado no agravamento de quadros (DE OLIVEIRA LG, et al., 2021). O primeiro caso confirmado da COVID-19, deu-se no dia 18 de março de 2020, passados um ano desde a primeira confirmação, até o dia 18 de março de 2021, mais de 393.675 casos foram notificados pela Secretaria de Saúde do Estado do Pará (SESPA), com uma variante de 9.682 mortes (SESPA, 2020).

Achados epidemiológicos são de grande relevância científica e fundamentais para a identificação dos fatores clinico-epidemiológicos relacionados à progressão da infecção e implementação de intervenções (DE OLIVEIRA LG, et al., 2021). À vista disso, faz-se necessário a compreensão sobre a COVID-19 no estado do Pará e o impacto por ela causado. Para tanto, o objetivo do estudo é descrever as evoluções e repercussões de um ano após o primeiro caso de SARS-CoV2 no Estado do Pará, Brasil.

\section{MÉTODOS}

Trata-se de um estudo epidemiológico-descritivo dos casos confirmados e óbitos por COVID-19 no Estado do Pará, no período de 18 de março de 2020 (data do primeiro caso confirmado no estado) até 18 de março de 2021.

O Estado do Pará pertence à região Norte do Brasil e é considerado o segundo maior estado do país em extensão territorial, de aproximadamente $1.245 .870,707 \mathrm{~km}^{2}$, contendo uma população estimada de 8.690.745 pessoas. A região faz fronteira com os estados Amapá, Maranhão, Tocantins, Mato Grosso e Roraima, e com os países Suriname e Guiana; além de abrigar uma larga faixa da floresta Amazônica (IBGE, 2021).

Os dados foram extraídos através da plataforma da Secretaria do Estado do Pará (SESPA), com dados disponibilizados no período de 2020 a 2021, incluindo todos os casos confirmados de infecção pelo novo coronavírus em residentes do estado divulgados pela plataforma. 
Foram coletados dados do site da Secretaria Estadual de Saúde (SESPA), site informativo que realiza diariamente atualizações sobre o monitoramento dos casos de COVID-19 no Estado. Foram consideradas as seguintes variáveis: Sexo, faixa etária, internação, comorbidades, sintomas, número de leitos e testes aplicados nos casos confirmados e óbitos. Os dados foram organizados, tabulados e analisados por meio do software Microsoft Office Excel $2013^{\circledR}$, no qual foram aplicadas análises estatísticas descritivas e a produção de tabelas e gráficos.

Por se tratar de dados epidemiológicos de um banco de domínio público, a pesquisa não precisou da avaliação do Comitê de Ética em Pesquisa. Estando de acordo com a resolução no 510 de 07 de abril de 2016, do Conselho Nacional de Saúde que disciplina pesquisas realizadas com seres humanos e com os termos da Lei $\mathrm{n} \cong 12.527$ de 18 de novembro de 2011 que regulamenta pesquisas com informações de acesso e domínio público (BRASIL, 2016; BRASIL, 2011).

\section{RESULTADOS}

Foram analisados todos os casos confirmados entre 18/03/2020 e 31/03/2021, o que corresponde ao panorama de um ano de COVID-19 no Estado do Pará, ao qual foram registrados 420.372 casos pela SESPA.

Na Figura 1, pode-se observar os casos confirmados e óbitos por mês. Dessa forma, em maio de 2020 foi registrado o maior índice de casos confirmados 17,05\% ( $\mathrm{N}=73.739)$ e óbitos com o índice de $29,7 \%$ $(\mathrm{N}=3.121)$.

Nota-se que o mês de outubro de 2020 foi o mês com menos casos confirmados, com uma taxa de 3,7\% $(\mathrm{N}=15.817)$. Enquanto nos óbitos, o mês de março de 2020 , houve $0,009 \%(\mathrm{~N}=1)$ por decorrência que foi a primeira morte por COVID-19 no estado do Pará, logo após o pico de óbitos no mês de maio de 2020 o menor índice foi $1,9 \%(N=191)$ no mês de outubro.

Podemos observar ainda no gráfico, que tanto nos casos confirmados quanto nos óbitos houve um declínio após o pico do mês de maio de 2020, possivelmente em razão dos protocolos de isolamento e a efetivação por parte da população, desta forma ocorreu uma diminuição da ocorrência de transmissão durante esse período.

Foi observado no período de março de 2021 que houve um acréscimo tanto dos casos confirmados quanto os de óbitos, sendo assim acarretando uma possível segunda onda de contágio pelo vírus da COVID-19.

Figura 1 - Evolução do número de casos e óbitos confirmados em um ano de COVID-19, no Estado do Pará, 2021.

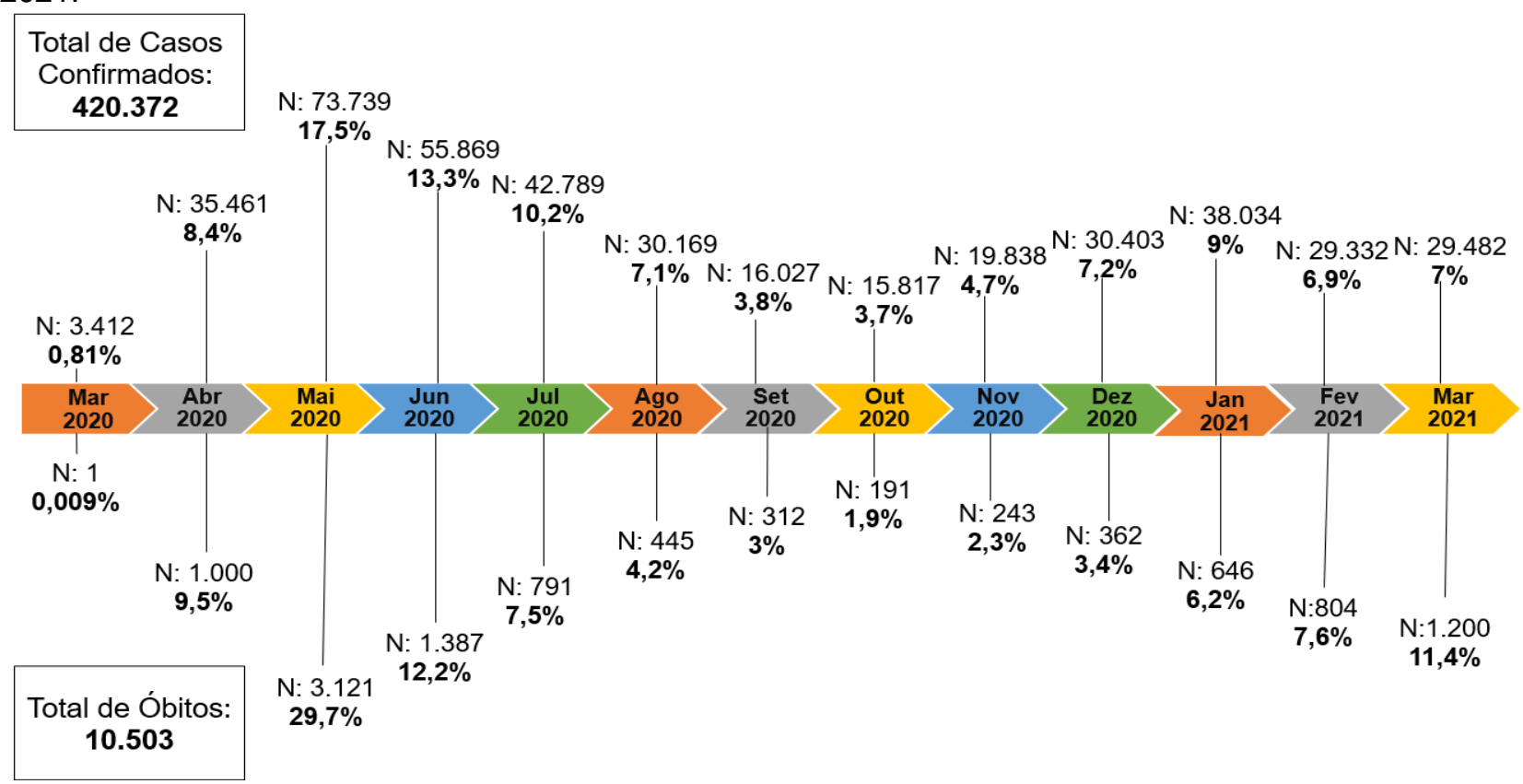

Fonte: Matos MJJ, et al., 2021; dados extraídos de Secretaria de Saúde do Estado do Pará (SESPA), 2021. 
De acordo com a Tabela 1 foram analisados 396.302 casos, onde $53,4 \%(211.625 / 396.302)$ eram do sexo feminino. Observou-se na população examinada maior frequência na faixa etária de 30 a 39 anos, a qual corresponde a $23,5 \%$ (93.058/396.302) e a menor frequência foi identificada entre os maiores de oitenta anos $2,1 \%(8.531 / 396.302)$. A Tabela 1 ainda especifica que 1,1\% (4.339/396.302) foram ignorados, ou seja, encontraram-se sem essas informações.

Tabela 1 - Distribuição de casos confirmados da COVID-19 por faixa etária e sexo, Estado do Pará, 2021.

\begin{tabular}{lcccccc} 
Faixa etária & \multicolumn{2}{c}{ População } & \multicolumn{4}{c}{ Sexo } \\
\cline { 4 - 7 } (anos) & $\mathbf{N}$ & $\%$ & Masculino & $\%$ & Feminino & $\%$ \\
\hline 0 a 9 & 17.498 & 4,4 & 8.727 & 49,8 & 8.771 & 50,1 \\
10 a 19 & 26.710 & 6,8 & 11.530 & 43,1 & 15.180 & 56,8 \\
20 a 29 & 67.771 & 17,1 & 30.120 & 44,4 & 37.651 & 55,5 \\
30 a 39 & 93.058 & 23,5 & 44.241 & 47,5 & 48.817 & 52,4 \\
40 a 49 & 76.279 & 19,3 & 35.587 & 46,6 & 40.692 & 53,3 \\
50 a 59 & 52.075 & 13,1 & 23.889 & 45,8 & 28.186 & 54,1 \\
60 a 69 & 32.836 & 8,3 & 15.611 & 47,5 & 17.225 & 52,4 \\
70 a 79 & 17.205 & 4,3 & 8.547 & 49,6 & 8.658 & 50,3 \\
> 80 anos & 8.531 & 2,1 & 4.133 & 48,4 & 4.398 & 51,5 \\
lgnorado & 4.339 & 1,1 & 2.292 & 52,8 & 2.047 & 47,1 \\
\hline Total & $\mathbf{3 9 6 . 3 0 2}$ & $\mathbf{1 0 0}$ & $\mathbf{1 8 4 . 6 7 7}$ & $\mathbf{4 6 , 6}$ & $\mathbf{2 1 1 . 6 2 5}$ & $\mathbf{5 3 , 4}$ \\
\hline
\end{tabular}

Fonte: Matos MJJ, et al., 2021; dados extraídos de Secretaria de Saúde do Estado do Pará (SESPA), 2021.

De acordo com a Tabela 2, pode-se observar que a maior concentração de óbitos é do sexo masculino $60,1 \%(N=6.310)$. Levando em consideração, que a maior concentração de óbitos na faixa etária do sexo masculino foi de 70 a 79 anos $26,7 \%(\mathrm{~N}=2.800)$, enquanto o feminino foi $10,4 \%(\mathrm{~N}=1.093)$. A menor frequência foi de 10 a 19 anos $0,5(\mathrm{~N}=50)$.

Tabela 2 - Distribuição de óbitos confirmados por faixa etária e sexo, Estado do Pará, 2021.

\begin{tabular}{ccccccc}
\hline Faixa etária & \multicolumn{2}{c}{ População } & \multicolumn{5}{c}{ Sexo } \\
\cline { 5 - 7 } (anos) & $\mathbf{N}$ & $\%$ & Masculino & $\%$ & Feminino & $\%$ \\
\hline $0-9$ & 56 & 0,5 & 25 & 0,2 & 31 & 0,3 \\
$10-19$ & 50 & 0,5 & 31 & 0,3 & 19 & 0,2 \\
$20-29$ & 138 & 1,3 & 81 & 0,8 & 57 & 0,5 \\
$30-39$ & 395 & 3,8 & 209 & 2 & 186 & 1,8 \\
$40-49$ & 759 & 7,2 & 496 & 4,7 & 263 & 2,5 \\
$50-59$ & 1.361 & 13 & 846 & 8 & 515 & 4,9 \\
$60-69$ & 2.452 & 23,3 & 1.510 & 14,4 & 942 & 9 \\
$70-79$ & 2.800 & 26,7 & 1.707 & 16,2 & 1.093 & 10,4 \\
80 ou mais & 2.492 & 23,7 & 1.405 & 13,3 & 1.087 & 10,3 \\
\hline Total & $\mathbf{1 0 . 5 0 3}$ & $\mathbf{1 0 0}$ & $\mathbf{6 . 3 1 0}$ & $\mathbf{6 0 , 1}$ & $\mathbf{4 . 1 9 3}$ & $\mathbf{3 9 , 9}$
\end{tabular}

Fonte: Matos MJJ, et al., 2021; dados extraídos de Secretaria de Saúde do Estado do Pará (SESPA), 2021.

Na Tabela 3 observa-se em ordem crescente a relação dos municípios do Estado do Pará com mais casos confirmados de COVID-19. O Pará é dividido em 144 municípios, e conforme a tabela, pode-se analisar os 
municípios com mais casos confirmados, como por exemplo a capital paraense, Belém, que possui maior índice com 20,45\%. Seguida por Parauapebas (8,84\%) e a região metropolitana de Belém, como a cidade de Ananindeua com índice de 3,89\%.

Tabela 3 - Municípios com mais casos confirmados pela COVID-19, Estado do Pará, 2021.

\begin{tabular}{ccc}
\hline Município & Confirmados & $\%$ dos casos \\
\hline Belém & 85,958 & $20,45 \%$ \\
Parauapebas & 37,157 & $8,84 \%$ \\
Ananindeua & 16,348 & $3,89 \%$ \\
Santarém & 16,186 & $3,85 \%$ \\
Marabá & 13,863 & $3,30 \%$ \\
Altamira & 10,778 & $2,56 \%$ \\
Redenção & 9,138 & $2,17 \%$ \\
Itaituba & 8,133 & $1,93 \%$ \\
Barcarena & 7,990 & $1,90 \%$ \\
Canaã dos Carajás & 6,902 & $1,64 \%$
\end{tabular}

Fonte: Matos MJJ, et al., 2021; dados extraídos de Secretaria de Saúde do Estado do Pará (SESPA), 2021.

As principais comorbidades relacionadas ao COVID-19 no estado do Pará são ilustradas no Gráfico 1, onde pode-se observar que as cardiopatias (43\%), seguido de diabetes $(33 \%)$ possuem os maiores índices de ocorrência, com 16.835 e 12.688 casos, respectivamente. Já as doenças neurológicas e a imunodeficiência possuem as menores taxas encontradas, com 1\% (496) e $3 \%$ (1.337) respectivamente.

Gráfico 1 - Levantamento das principais comorbidades da COVID-19, no Estado do Pará, 2021.

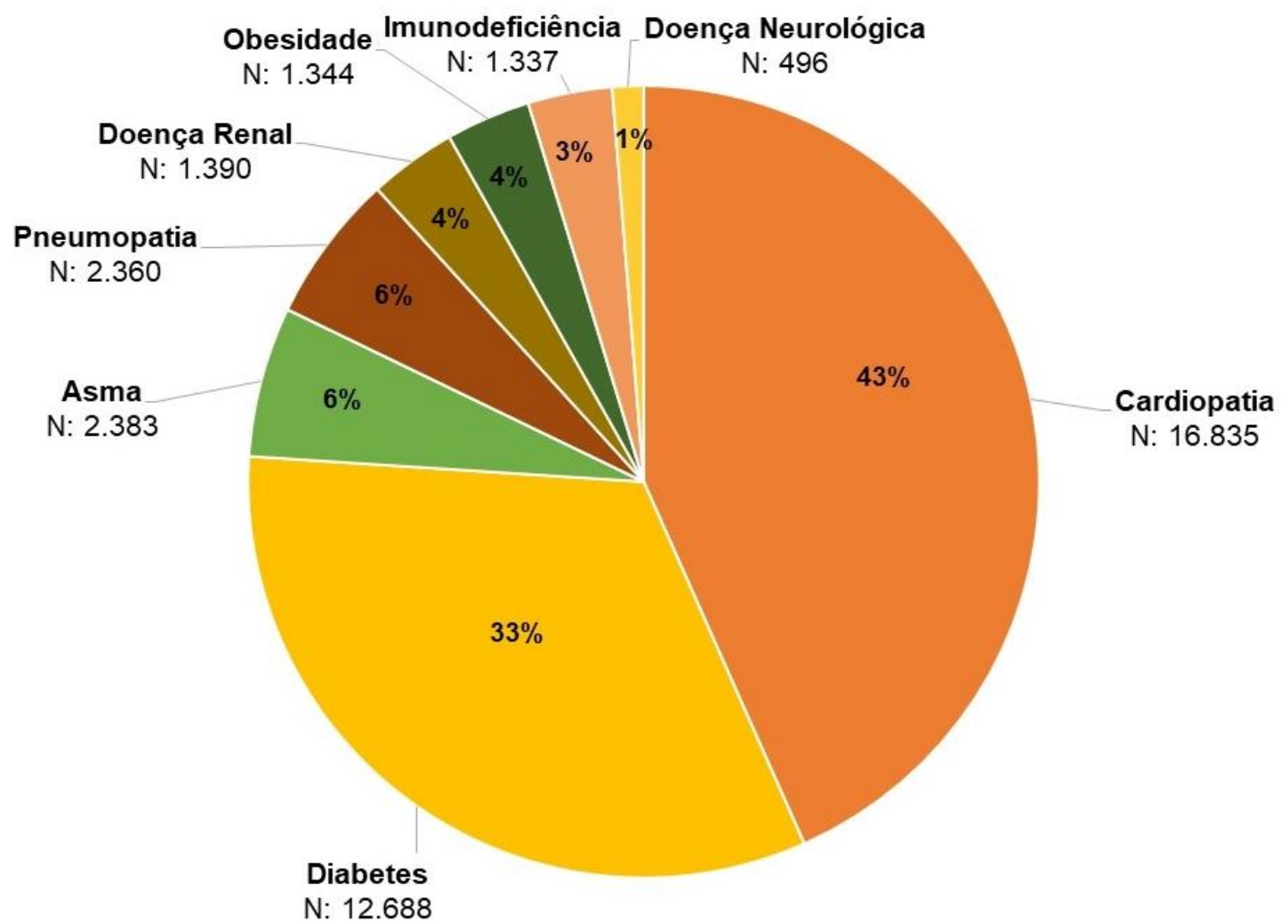

Fonte: Matos MJJ, et al., 2021; dados extraídos de Secretaria de Saúde do Estado do Pará (SESPA), 2021. 
Durante o período analisado, 791.668 testes foram aplicados. O teste rápido mostrou o maior percentual de realização, com $77,5 \%$. Os testes laboratoriais - PCR, por sua vez, apresentaram $22,5 \%$. Representando a maior frequência, para 613.251 de testes rápidos aplicados, 63,5\% resultaram como negativo. Enquanto para o teste laboratorial - Polymerase Chain Reaction (PCR), de 178.417 testes aplicados, 54,1\% tiveram resultado positivo. O teste rápido foi também utilizado nos critérios de casos confirmados em $69,59 \%$. Destaca-se ainda que o critério epidemiológico também foi utilizado, embora em menor frequência (6,56\%). Já para os casos de óbitos, o critério principal era o laboratorial em 57,50\%, conforme ilustra a Figura 2.

Figura 2 - Distribuição dos critérios de confirmação dos casos confirmados e óbitos por COVID-19, no Estado do Pará, 2021.

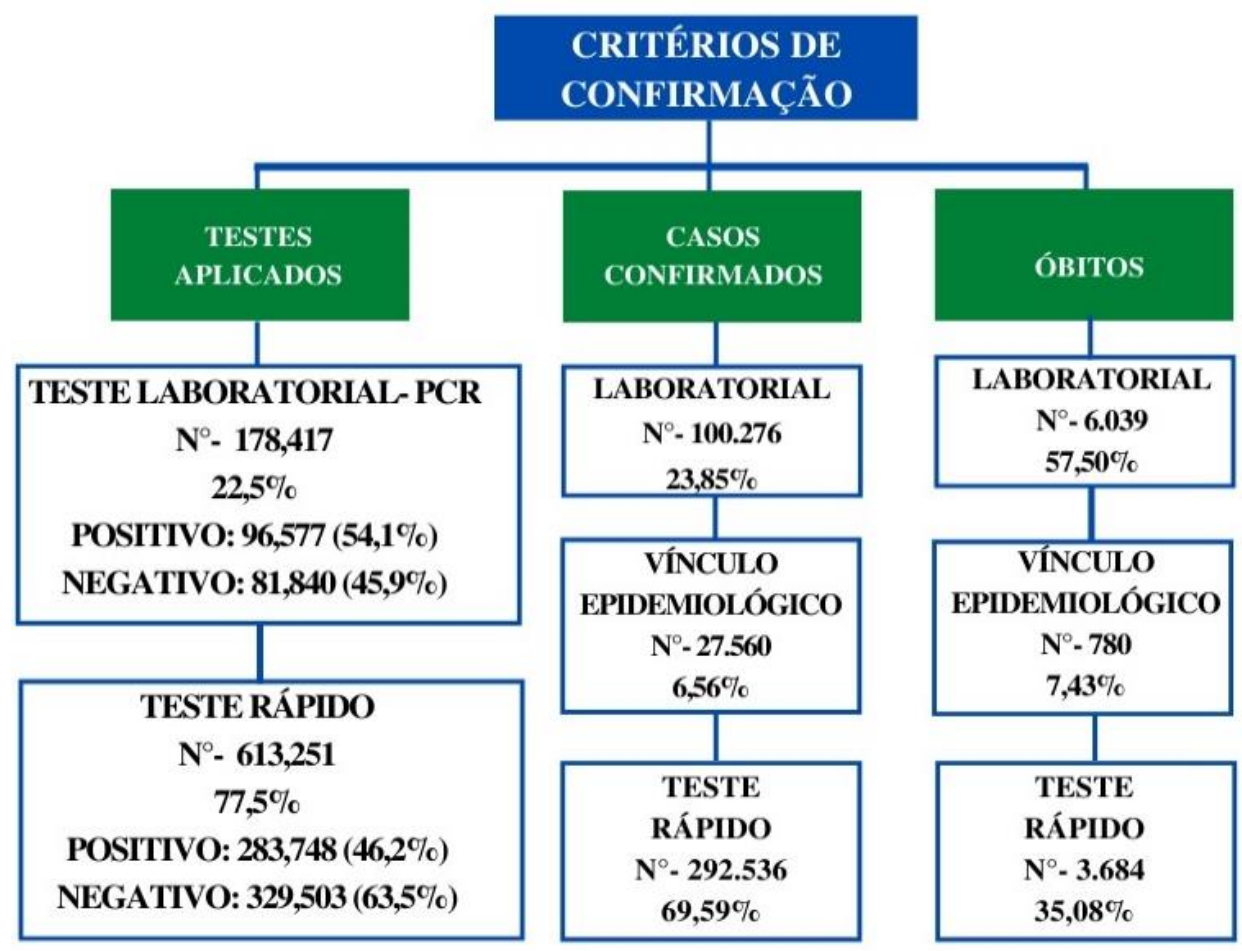

Fonte: Matos MJJ, et al., 2021; dados extraídos de Secretaria de Saúde do Estado do Pará (SESPA), 2021.

Em relação à clínica dos pacientes associados ao diagnóstico de COVID-19 no Estado do Pará, houve maior ocorrência de febre em 23\% (250.373), seguido de tosse em 22\% (238.101) e dor de garganta com $15 \%$ (164.658) dos casos. Os de menores frequências foram náusea/vômito em 1\% (16.924) e diarreia com $3 \%$ (37.817). No decorrer do ano, foi acrescentado diferentes sintomas como novas variáveis como: dispneia, mialgia, artralgia e coriza.

Enquanto aos leitos exclusivos para o COVID-19 no estado do Pará ao longo do ano, as Unidades de Terapia Intensiva Adulto (UTI adulto) não variaram muito, estando recorrente as altas taxas de ocupação, no momento da coleta apresentava um índice de 87,63\%, seguida dos leitos clínicos em ocupação de 80,41\%. A UTI Pediátrico encontrava-se em 58,33\% e o clínico pediátrico em 42,86\%, e em contrapartida a Unidade de Terapia Intensiva Neonatal encontrava-se com 100\% dos seus leitos disponíveis.

\section{DISCUSSÃO}

A pandemia causada pelo coronavírus tem gerado demanda de estratégias emergenciais e ações coletivas em prol de solucionar o problema pandêmico. Na Figura 1, observa-se que em maio de 2020 foi registrado o maior índice de casos confirmados. Em consequência, houve o cumprimento de determinações mais rigorosas, no intuito de conter o avanço da doença, sendo decretado lockdown entre as datas de 7 a 24 de maio, na capital Belém e outros municípios, permitido apenas a execução de atividades consideradas essenciais (VILHENA FDM et al., 2020). 
Diante disso, é visível a vulnerabilidade da população em relação às questões biológicas, físicas, econômicas e sociais. As variáveis que propiciam populações subdesenvolvidas à maior infecção por COVID19 são muitas, como por exemplo: uso de transporte público, número de moradores por residência, o baixo acesso ao saneamento básico e aos sistemas de saúde. Além disso, têm-se ainda, a problematização em relação ao isolamento social sem que haja perda monetária de renda, principalmente aos trabalhadores informais (CÂMARA SF, et al., 2021; FIGUEIREDO AM et al., 2020).

A velocidade da propagação do vírus e suas consequências, afetou a vida das pessoas e expôs as fragilidades dos sistemas de saúde. No Brasil em até 31 de maio de 2020, foram notificados 29.314 óbitos pela doença no país, por sua vez, no estado do Pará até o dia 31 de maio de 2020 chegou a 17,05\% dos casos, a determinação dos óbitos foram as condições prévias à entrada no sistema de saúde, associada às comorbidades relacionadas à COVID-19 (MACIEL EL, et al., 2020).

De acordo com os resultados descritos e apresentados na Tabela 1, observa-se a predominância de casos confirmados de COVID-19 em pessoas do sexo feminino 53,4 \%, apesar de não haver grande diferença em relação ao sexo masculino que foi de $46,6 \%$. Ademais, em análise comparativa, um estudo realizado no Nordeste revela que no Estado do Ceará o percentual de diagnósticos positivos se manteve no sexo masculino cerca 54,6 \% em relação ao sexo feminino 45,4\% (SESPA, 2021; DA SILVA RP, et al., 2020).

Em relação à faixa etária, de acordo com os dados da Secretaria de Saúde do Estado do Pará, houve uma predominância na faixa etária de 30 a 39 anos. De Oliveira LG, et al. (2021) categorizou os casos iniciais da COVID-19 no Estado do Pará e também destacou essa mesma faixa etária, evidenciando que não houve mudança significativa quanto à faixa etária nos primeiros casos até um ano de COVID-19 no Estado. Dessa forma, observa-se que a população economicamente ativa é a que possuem mais probabilidade de contrair o vírus por passarem a maior parte do tempo fora de seu ambiente domiciliar, diferente da faixa etária menos acometidas a qual permanece a maior parte do tempo dentro do seu domicílio, principalmente, pela suspensão das aulas presenciais, diminuindo assim o contato dessa faixa etária com outros indivíduos (ARAÚJO AAC, et al., 2020).

Conforme pode ser visto na Tabela 2, no Pará o coeficiente de mortalidade pelo vírus é maior entre os homens em relação às mulheres. Em relação à taxa de mortalidade por faixa etária mostrou-se especificamente no grupo da terceira idade $26,7 \%$ entre 70 a 79 anos, os idosos são destaque na pandemia SARS-CoV-2 a grande parte por apresentar alterações decorrentes da senescência ou senilidade (MACHADO CJ, et al., 2020).

A população do Norte do Brasil, especialmente o estado do Pará, quando comparada aos demais estados brasileiros, não possui as mesmas condições de acesso aos serviços de saúde e saneamento básico. Por esses fatores, quando relacionado a países desenvolvidos como a Austrália, que até hoje quantificou cerca de 29.897 casos. Esse número quando comparado ao estado do Pará é uma variante de somente $7 \%$ dos casos do estado, e conforme é ilustrado na Tabela 3, somente a capital Belém já supera o índice da Australia, pois só no município registra-se mais de 85.958 casos confirmados (SILVA TTM et al., 2019; AUSTRÁLIA, 2021; SESPA, 2021).

Outrossim, o quadro sintomatológico da COVID-19 averiguado nesta pesquisa, é parecido ao que é encontrado em outros estudos da literatura (LIU X et., 2020). Sabe-se que, a manifestação sintomatológica do novo coronavírus é similar aos sintomas da gripe, com febre, tosse, dor de garganta, com potencial de manifestação de dispneia, fadiga, mialgia e cefaleia. Pesquisa realizada na China, identificou nos pacientes da região a existência de sintomatologia respiratória, como por exemplo tosse (76\%), dispneia (55\%) e presença de febre (98\%) (BRASIL, 2020; HUANG C, et al., 2020).

A cardiopatia, com base no Gráfico 1, configura-se como a principal comorbidade ou fator de risco para morte por COVID-19 no estado. E quando relacionada ao fator idade, tende-se aos riscos de morte serem mais acentuados. Pois, sabe-se que no Brasil, há uma crescente mudança na pirâmide etária, pelo aumento percentual da população idosa, gerando um predomínio de Doenças Crônicas Não Transmissíveis (DCNT), 
dentre esses distúrbios, o desenvolvimento de doenças cardiovasculares - com as cardiopatias - que se constituem como a comorbidade que mais causa mortes mundialmente (SILVA TTM et al., 2019; SIMÃO LTSS, et al., 2019).

Não obstante, mesmo com a diversidade indicada nos índices de testes aplicados na população, a pesquisa apresenta resultados que identificam que a maioria da população paraense não testa o bastante para determinar de maneira adequada os indicadores de saúde necessários relacionados ao novo coronavírus. Conforme demonstrado na Figura 2, somente 791.668 pessoas realizaram testes, e esse valor representa apenas $9 \%$ da população geral, visto que há mais de oito milhões de pessoas que residem no estado do Pará. Dessa maneira, com base nos baixos índices de testes, pode haver, como resultados, a desconsideração dos números reais de indivíduos infectados pela COVID-19 (IBGE, 2021; PILECCO FB, et al., 2021).

Com o pico do vírus no mês de maio, conforme também é mostrado na Figura 2 houve um acarretamento do impacto nos hospitais que ofertavam os leitos, com índice da ocupação pelo leito da UTI adulto em até $87,63 \%$, dessa forma, tornou-se grande a sobrecarga de pessoas infectadas e espera de leitos disponíveis. A falência da atenção básica resultou no agravamento de quadros em situações que ainda eram passíveis de reversão sem necessidade de cuidados intensivos, dessa forma, sobrecarregando os leitos de UTIs disponíveis na rede (De OLIVEIRA LG, 2021).

Com os hospitais ficando sobrecarregados, o governo do estado do Pará percebeu a necessidade de implantar hospitais de campanha para a internação de pacientes infectados com a COVID-19. Foram disponibilizados 720 leitos clínicos e UTI em quatro regiões do estado (SESPA, 2021; PARÁ, 2020).

O Brasil apresenta desigualdades geográficas e desproporção de recursos para acesso dos leitos de internação de UTI, a concentração maior é nas regiões Sul e Sudeste do país. Diante dos dados e dos resultados do presente estudo, é de suma importância salientar o impacto das desigualdades em saúde na população mais vulnerável, com mais dificuldade para perceber a importância do autocuidado, o acesso aos serviços de saúde e aos níveis de maior complexidade no SUS tendo em vista o seu direito adquirido (CONTE D, et al., 2020; MACIEL EL, et al., 2020).

Em suma, vale ressaltar as limitações do presente estudo em relação à coleta de dados, devido a atualização constante da plataforma de dados, semelhante as dificuldades encontradas por Vilhena (2020) que sugere a implementação de um sistema de informação unificado e ágil, possa ser uma estratégia para o enfrentamento da pandemia. Além disso, sabe-se que mesmo com o número alto de notificações por COVID19, estima-se que esses casos são 14 vezes maiores que os dados divulgados oficialmente (ALVES D, 2020).

Mesmo com a oscilação dos casos, há ainda índices significativos de infecções e mortalidade por COVID19. Dessa forma, demonstra-se a necessidade do monitoramento e definição de medidas específicas no estado, com políticas públicas adequadas, pois só assim haverá rumos positivos durante a pandemia, com atenção especial àqueles que precisam de suportes maiores, devido aos riscos e vulnerabilidades, como por exemplo aos idosos e crianças. Com base nisso, a produção desta evidência é de extrema importância para a comunidade científica nacional e internacional por apresentar particularidades de uma população amazônica do norte do Brasil em meio ao contexto histórico e social da humanidade.

\section{CONCLUSÃO}

Diante do exposto, acredita-se que com a identificação e esclarecimento dos dados epidemiológicos acerca da COVID-19, possibilita-se que haja uma reflexão sobre as condições e notificações dos casos no estado, no qual é causado pelas subnotificações, sendo eficaz em retratar os índices de morbidade e mortalidade, divulgando variáveis que influenciam nos processos de tratamento da saúde pública referente a realidade da pandemia. Ademais, os dados podem contribuir para um melhor entendimento social e clínico, podendo ser considerados como elementos importantes a serem analisados, estimulando a preocupação com os cuidados e medidas preventivas, bem como os conhecimentos sobre a patologia. 


\section{REFERÊNCIAS}

1. ALVES D, et al. Estimativa de casos de Covid-19. Covid-19 Brasil: monitoramento e análises da situação do Coronavírus no Brasil. São Paulo, 2020.

2. ARAÚJO AAC, et al. COVID-19: analysis of confirmed cases in Teresina, Piaui, Brazil. Revista Prevenção de Infecção e Saúde. 2020, 6.

3. AUSTRÁLIA. Coronavirus Pandemic (COVID-19). 2021. Disponível em: https://ourworldindata.org/coronavirus-data

4. BRASIL. Ementa Constitucional oㅜ 12.527, de 18 de janeiro de 2011. Lei №12.527, de 18 de novembro de 2011.2011. Disponível em: http://www.planalto.gov.br/ccivil_03/_ato2011-2014/2011/lei/l12527.htm

5. BRASIL. Ministério da Saúde. Coronavírus: COVID-19 [Internet]. 2020. Disponível em: https://www.gov.br/saude/ptbr

6. BRASIL. Ministério da Saúde. Diário Oficial da União. RESOLUÇÃO № 510, DE 7 DE ABRIL DE 2016. 2016. Disponível em: https://bvsms.saude.gov.br/bvs/saudelegis/cns/2016/res0510_07_04_2016.html

7. CÂMARA SF, et al. Socioeconomic vulnerability of communities on the Brazilian coast to the largest oil spill (20192020) in tropical oceans. Ocean \& Coastal Management. 2020, 202: 105506.

8. CAMPOS ACV, LEITÃO LPC. Letalidade da COVID-19 entre profissionais de saúde no Pará, Brasil/Lethality of COVID19 among healthcare professionals in Pará, Brazil/Letalidad de la COVID-19 entre profesionales de la salud en Pará, Brasil. Journal Health NPEPS. 2020, 6(1).

9. CONTE D, et al. Oferta pública e privada de leitos e acesso aos cuidados à saúde na pandemia de COVID-19 no Brasil. Saúde em Debate, 2020, 1(26)

10. DA SILVA RP, et al. Cenário epidemiológico da COVID-19 no nordeste do Brasil. Saúde Coletiva (Barueri), 2020,10 (59): 4420-4429.

11. DE OLIVEIRA LG, et al. Análise do índice inicial de casos de Covid-19 relacionado os indicadores sociais de saúde no estado do Pará, Brasil. Revista Eletrônica Acervo Saúde, 2020, 13(2).

12. FIGUEIREDO AM, et al. Social determinants of health and COVID-19 infection in Brazil: an analysis of the pandemic. Revista brasileira de enfermagem, 2020, 73.

13. FRANÇA EB, et al. Óbitos por COVID-19 no Brasil: quantos e quais estamos identificando? Revista Brasileira de Epidemiologia, 2020, 23: 200053.

14. IBGE. Instituto Brasileiro de Geografia e Estatística. Pará. Pará: Panorama. 2021.

15. HENRIQUE MC, LINHARES AMA. Marajoara Pottery and Círio de Nazaré: meaning and consecration of the Brazilian cultural heritage. Topoi (Rio de Janeiro), 2019, 20(41): 394-420.

16. HUANG C, et al. Clinical features of patients infected with 2019 novel coronavirus in Wuhan, China. Lancet, 2020, 395(10223): 497-506.

17. LIMA FET, et al. Intervalo de tempo decorrido entre o início dos sintomas e a realização do exame para COVID-19 nas capitais brasileiras, agosto de 2020. Epidemiologia e Serviços de Saúde, 2020, 30: 2020788.

18. LIU X, et al. COVID-19: Progress in diagnostics, therapy and vaccination. Theranostics, 2020,10(17): 7821.

19. MACHADO CJ, et al. Estimativas de impacto da COVID-19 na mortalidade de idosos institucionalizados no Brasil. Ciência \& Saúde Coletiva, 2020, 25: 3437-3444.

20. MENDONÇA FD, et al. Região Norte do Brasil e a pandemia de COVID-19: análise socioeconômica e epidemiológica/North region of Brazil and the COVID-19 pandemic: socioeconomic and epidemiologic analysis/Región Norte de Brasil y la pandemia de COVID-19: análisis. Journal Health Npeps, 2020, 5(1): 20-37.

21. MACIEL EL, et al. Fatores associados ao óbito hospitalar por COVID-19 no Espírito Santo, 2020. Epidemiol. Serv. Saúde, Brasília, 2020, 29(4): 1-11.

22. OLIVEIRA WK, et al. Como o Brasil pode deter a COVID-19. Epidemiologia e Serviços de Saúde, 2020, $29: 2020044$.

23. PILECCO FB, et al. O efeito da testagem laboratorial nos indicadores de acompanhamento da COVID-19: uma análise dos 50 países com maior número de casos. Epidemiol. Serv. Saúde, Brasília, 2020, 30: 2.

24. SESPA. Secretária de Saúde Pública do Estado do Pará. Coronavírus no Pará (portal). 2020. Disponível em: http://www.saude.pa.gov.br/category/nota-informativa-covid-19/

25. SESPA. Secretária de Saúde Pública do Estado do Pará. Coronavírus no Pará (portal). 2021. Disponível em: http://www.saude.pa.gov.br/coronavirus/

26. SILVA TTM, et al. Pacientes submetidos à angioplastia transluminal coronariana: análise epidemiológica e angiográfica. Rev. Enferm, 2019, 10(3).

27. SILVA LÁN, et al. Acesso e acolhimento na Atenção Básica da região Oeste do Pará. Saúde em Debate, 2020, 43: 742-754.

28. SIMÃO LTSS, et al. Perfil dos idosos com doenças crônicas não transmissíveis internados em unidade de terapia intensiva. Rev. Enferm. 2019, 10(1).

29. SOARES KHD, et al. Medidas de prevenção e controle da covid-19: revisão integrativa. Revista Eletrônica Acervo Saúde, 2021, 3(2): 6071-6071.

30. VILHENA FDM, et al. Epidemic Curve of Covid-19 Cases after Lockdown in the Municipality of Belém, Pará State, Brazil. International Journal of Tropical Disease e Health, 2020: 6-13. 